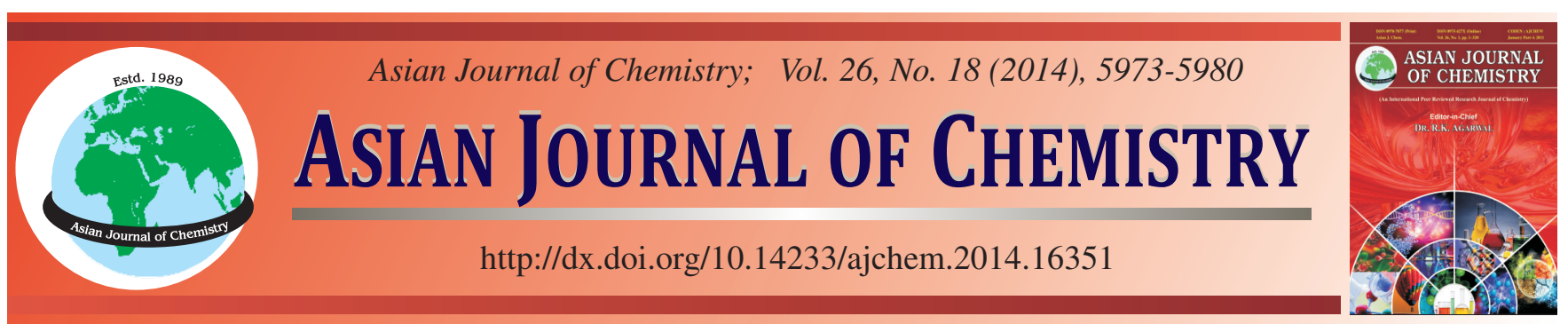

\title{
Synthesis and Electropolymerization of New Phenylene-Substituted Dipyrridyls: Electrochemical and Spectroscopic Characterization
}

Abdou Karim Diagne Diaw ${ }^{1}$, Modou Fall ${ }^{1, *}$, Diariatou Gningue-Sall ${ }^{1}$, Jean-Jacques Aaron $^{2}$ and Abderrahim Yassar $^{3}$

${ }^{1}$ Laboratoire de Chimie Physique Organique et d'Analyses Environnementales (LCPOAE), Département de Chimie, Faculté des Sciences et Techniques, Université Cheikh Anta Diop, BP 5005, Dakar-Fann, Sénégal

${ }^{2}$ Université Paris-Est, Laboratoire Géomatériaux et Environnement (EA 4508), UPEMLV, 77454 Marne-la-Vallée, France

${ }^{3}$ LPICM (UMR 7647), Ecole Polytechnique, Route de Saclay, 91128 Palaiseau, France

*Corresponding author: E-mail: modou.fall@ucad.edu.sn

\begin{abstract}
The present paper sets out the synthesis and electropolymerization process of 1,1'-phenylenedipyrridyl and 1,1'-diphenylenedipyrridyl. These compounds are prepared by Clauson-Kaas methods with 35 or $71 \%$ yield and characterized. UV-visible electronic absorption spectra and electrochemical methods were used to examine the effects of the electronic $\pi$-system extension in $1,1^{\prime}$-phenylenedipyrridyl and 1,1'-diphenylenedipyrridyl structures on the monomer properties relative to N-phenylpyrrole. Also, 1,1'-phenylenedipyrridyl and $1,1^{\prime}$-diphenylenedipyrridyl were electropolymerized on platinum electrode by anodic oxidation in $0.1 \mathrm{M}$ tetrabutylammonium hexafluorophosphate $\left(\mathrm{Bu}_{4} \mathrm{NPF}_{6}\right)$ acetonitrile or dichloromethane solutions and characterized by electrochemical and spectroscopic techniques. The electronic $\pi$-system extension effects on the poly(1,1'-phenylenedipyrridyl) physico-chemical properties and structure were investigated by cyclic voltammetry, FT-IR, UV-visible and XPS spectroscopy and scanning electron microscopy (SEM). Cyclic voltammetry of the poly(1,1'-phenylenedipyrridyl) films indicated a good redox behaviour and an improvement of the electrochemical properties. FT-IR spectral studies indicated a linear structure of this polymer with 2, 5 coupling on the pyrrole ring. SEM analysis showed that polymer film morphology changed between the oxidized and reduced states. The solid-state UV-visible spectra of the poly(1,1'phenylenedipyrridyl) films on ITO were characterized by a significant red-shift of the absorption maximum, as compared to poly(1,1'phenylenedipyrridyl) film. Moreover, the very low amount of poly(1,1'-diphenylenedipyrridyl) film obtained during the electropolymerization process and electrochemical studies of electrosynthesized poly(1,1'-diphenylenedipyrridyl) showed that the polymer properties modulated by extension of the monomer electronic $\pi$-system can be limited by steric effects.
\end{abstract}

Keywords: Synthesis, Phenylpyrrole and derivatives, Electropolymerization, Electrocatalytic effect.

\section{INTRODUCTION}

Pyrrole is one of the most used heterocyclic compounds because of the increasing interest which it arouses regarding medicnal chemistry, organic synthesis and chemistry of materials ${ }^{1-7}$. Consequently, various synthesis procedures were developed for pyrrole and its derivatives. Among these procedures, we can quote the following classic methods: (i) Hantzsch reaction $^{8,9}$, which allows one to obtain pyrrole from the reaction between $\alpha$-chloromethyl ketone, $\beta$-ketoester and ammonia water. (ii) Knorr reaction ${ }^{10-14}$, leading to pyrrole by reaction between $\alpha$-aminoketone and $\beta$-ketoester. (iii) Paal Knorr reaction ${ }^{15}$ which allows to obtain pyrrole by condensation of 2,5-dimethoxytetrahydrofuran and one primary amine.

The interest of monomers stemming from these diverse modes of synthesis made of the chemistry of materials a much widened domain where organic chemists, electrochemists and photochemists go alongside ${ }^{17-19}$. This enthusiasm is partly related to the possibility of modifying the monomeric unit by grafting of numerous chemical functions, which often leads to a modification of the various properties ${ }^{20-22}$. To this optimization is added the possibility of polymerizing these monomers in various media (organic, micellar, etc.) by means of several techniques (electrochemical, chemical, etc.) and, finally, of utilizing the obtained polymers for several applications.

In previous works, the authors studied the impact of the electrosynthesis environment and the electrocatalytic effect of pyrrole which has a lower oxidation potential on the Nphenylpyrrole (N-PhPy) $)^{24-28}$ electropolymerization process. So, it turned out that the presence of small amounts of pyrrole and the optimization of the environment could lead to an improvement of the electrosynthesized poly(N-phenylpyrrole) physicochemical properties ${ }^{26,27}$. We showed that the use of micellar media for the electropolymerization of the $\mathrm{N}$-phenylpyrrole 
could bring many advantages such as the decrease of the oxidation potential and the increase of the polymer solubility ${ }^{27}$. Nevertheless, we also revealed in these works some blockings related to the insolubility of poly(N-phenylpyrrole) films obtained in organic medium ${ }^{26}$ and to problems of films overoxidation and degradation due to the use of perchloric acid in aqueous micellar medium ${ }^{16}$. To circumvent these difficulties, we realized afterward other studies centered on the synthesis, characterization and analysis of the functional properties of a new series of $p$-substituted $\mathrm{N}$-phenylpyrroles for the preparation of new polymers ${ }^{28-30}$. The introduction of electrondonating substituents or the extension of the $\pi$-system, by grafting of pyrrole and/or phenyl on the N-phenylpyrrole skeleton, should present a big interest for the electrochemical preparation of new polymer materials, intended to applications as organic components having original electrochemical and optical characteristics. We had already shown that the presence of these electron-donating substituents, could significantly improve the N-phenylpyrrole electrochemical and spectroscopic properties ${ }^{28}$. These changes led to important red-shifts of the maximum absorption wavelength with a significant increase of the molar absorption coefficient value on the UVvisible spectra and a strong decrease of the oxidation potential. These phenomena are due to an increase in the chain-length resulting in the $\pi$-conjugated system extension. These observations led us to design new electroactive materials such as poly $\left(1,1^{\prime}-p\right.$-phenylenedipyrridyl) [poly(PhDPy)] or poly $\left(1,1^{\prime}-\right.$ $p$-diphenylenedipyrridyl) [poly(DPhDPy)] to appreciate the impact of the extent of the $\pi$-system with regard to the $\mathrm{N}$ phenylpyrrole on the physico-chemical properties of the corresponding polymer. In a recent paper ${ }^{30}$, we presented the electrosynthesis, electrochemical properties and characterization of these compounds, along with poly(N-p-hydroxyphenylpyrrole), poly(N-p-methoxyphenylpyrrole) and poly(N-p-thiophenephenylpyrrole).

The present paper focuses on the description of the organic synthesis of PhDPy and PhDPy by the Clauson-Kaas method ${ }^{31}$ which presents more advantages from the point of view of the reaction kinetics, yield and simplicity. While 1,1'-p-phenylenedipyrridyl has already been synthesized in the literature, we have not found any data for the second compound (except in our recent paper $^{30}$ ). Therefore, to the best of our knowledge, this is the first time that $1,1^{\prime}-p$-diphenylenedipyrridyl is obtained. We also studied the electrochemical and spectroscopic characteristics of the monomer and of the polymer, electrosynthesized in an organic medium. Moreover, we realized a comparative study of the influence of the pyrroleinduced electrocatalytic effect, whose oxidation potential is lower ${ }^{26}$ and the extent of the $\pi$-electronic system by grafting a second pyrrolic ring on $\mathrm{N}$-phenylpyrrole in para position with regard to the other pyrrole group in 1,1'-p-phenylenedipyrridyl, on the improvement of the obtained polymer properties.

\section{EXPERIMENTAL}

The following analytical-grade reagents and solvents were used in the synthesis process, the electropolymerization and the characterizations, without further purification: 2,5dimethoxytetrahydrofuran (Aldrich), $p$-phenylenediamine
(Avogadro), acetic acid (Aldrich), benzidine (Aldrich), naphthalene (Prolabo), 1,4-dioxane (sds), cyclohexane (sds), ethanol (Prolabo), acetonitrile (Aldrich), dimethylsulfoxide (Acros), dimethylformamide (ucb-Laboratoires Standa) and tetrabutylammonium hexafluorophosphate (Avogado).

General procedure: The electropolymerization was realized in an organic solvent $\left(\mathrm{CH}_{3} \mathrm{CN}\right.$ or $\left.\mathrm{CH}_{2} \mathrm{Cl}_{2}\right)$ in the presence of $10^{-2} \mathrm{M}$ of 1,1'- $p$-phenylenedipyrridyl (or $10^{-3} \mathrm{M}$ of 1,1'-diphenylenedipyrridyl) as monomer and $10^{-1} \mathrm{M}$ of $\mathrm{Bu}_{4} \mathrm{NPF}_{6}$ (electrolytic salt). The electrochemical measurements were made by means of an EG\&G Princeton Applied Research Model 362 Potentiostat/Galvanostat, coupled with a Kipp and Zonen type X-Y-t plotter. The study of the electropolymerization was realized by cyclic voltammetry, by impressed current density method $\left(0.125-2 \mathrm{~mA} \mathrm{~cm}^{-2}\right)$ and by voltage clamp method in a 3-collar flask connected with the potentiostat by three electrodes. We used a $5 \mathrm{~mm}$ diameter platinumdisk as working electrode, a stainless steel grid as counterelectrode and the saturated calomel electrode as the reference electrode (ECS: $+242 \mathrm{mV}$ with regard to Hydrogen Normal Electrode). The films were characterized by electrochemical and spectroscopic methods.

UV-visible spectra were recorded by means of a Lambda 2 Perkin Elmer UV-visible Spectrophotometer. FT-IR spectra were measured using a Nicolet S-60 Fourier Transform Infrared Spectrophotometer. X-Photoelectron Spectroscopy analysis was performed with a VG Instruments Escalab MK1 Photoelectron Spectrometer. The films morphology was studied by Scanning Electronic Microscopy (SEM) using a Leica Stere Scan 440 type scanning electron instrument.

\section{RESULTS AND DISCUSSION}

Synthesis of 1,1'-p-phenylenedipyrridyl and 1,1'diphenylenedipyrridyl: Synthesis and characterization of both compounds, namely 1,1'-p-phenylenedipyrridyl (PhDPy) and 1,1'-diphenylenedipyrridyl (DPhDPy), was performed as described in our previous paper ${ }^{28}$.

Preparation of 1, 1 '- $p$-phenylenedipyrridyl $(\mathrm{PhDPy})^{28}$ : In a $250 \mathrm{~mL}$ tri-collar flask containing $100 \mathrm{~mL}$ of glacial acetic acid, we introduce $50 \mathrm{mmol}$ of 2,5-dimethoxytetrahydrofurane $(6.46 \mathrm{~mL})$ and $25 \mathrm{mmol}(2.70 \mathrm{~g})$ of $p$-phenylenediamine. This mixture is heated in reflux until a precipitate is formed and 5 min after we add $50 \mathrm{~mL}$ of glacial acetic acid. After $15 \mathrm{~min}$ of heating, we add some carbon black to decolourize the reaction solution. Cool down and filter under vacuum. The filtrate is left to rest for $2 \mathrm{~h}$. We observe then a light brown powder which crystallizes and settles at the bottom. This product is then filtered and washed with acetic acid and ether. The condensation reaction of 2,5-dimethoxytetrahydrofuran and p-phenylenediamine in the ratio 1:2 (mol:mol) has led after 20-25 min to the formation of $1.87 \mathrm{~g} \mathrm{(36 \% )} \mathrm{of} \mathrm{1,1'-phenylene-}$ dipyrridyl (Scheme-I), with $\mathrm{T}_{\mathrm{f}}=229^{\circ} \mathrm{C}$ (literature: $228^{\circ} \mathrm{C}^{32}$ ).

${ }^{1} \mathrm{H}$ NMR (200 MHz, $\mathrm{CDCl}_{3}$ ): $\delta 7.08$ (app t, $J=2.1$ et 2.2 $\left.\mathrm{Hz}, 2 \mathrm{H} . \mathrm{H}_{2,5}\right), 6,4$ (app t, $J=2.1$ et $\left.2.2 \mathrm{~Hz}, 2 \mathrm{H}, \mathrm{H}_{3,4}\right), 7.4(\mathrm{~s}, 4 \mathrm{H}$, $\left.\mathrm{H}_{2,6^{\prime}, 3^{\prime}, 5^{\prime}}\right) .{ }^{13} \mathrm{C}$ NMR $\left(200 \mathrm{MHz}, \mathrm{CDCl}_{3}\right): \delta: 138.6\left(\mathrm{C}_{1}{ }^{\prime}, \mathrm{C}_{4}{ }^{\prime}\right), 121.5$ $\left(\mathrm{C}_{2}^{\prime}, \mathrm{C}_{3}{ }^{\prime}, \mathrm{C}_{5}{ }^{\prime}, \mathrm{C}_{6}{ }^{\prime}\right), 119.3\left(\mathrm{C}_{2,5}\right), 110.6\left(\mathrm{C}_{3,4}\right)$. FT-IR $\left(\mathrm{KBr}, \mathrm{cm}^{-1}\right)$ : 3125; 3109, 3073; 3047; 1581 ; 1526; 1472; 1482; 1433; 1388; $714 ; 1068 ; 1023 ; 641 ; 609 ; 619 ; 543$. Elemental analysis $(\%)$ : 


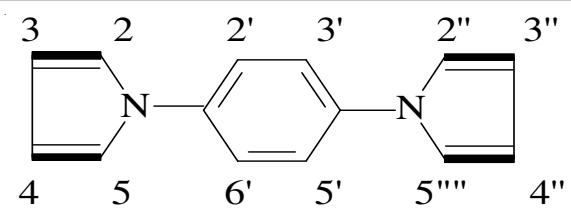

Scheme-I: Chemical formula of 1,1'-phenylenedipyrridyl

Calculated: C: $80.74, \mathrm{H}: 5.81, \mathrm{~N}: 13.45$; obtained: C: 80.17 ; H 5.80, N: 13.20.

Preparation of 1,1'-diphenylenedipyrridyl (DPhDPy) ${ }^{28}$ : Using the same procedure as for the previous product, the condensation reaction between $1.4 \mathrm{~mL}$ of 2,5 -dimethoxytetrahydrofuran $(0.010 \mathrm{~mol})$ and $4.60 \mathrm{~g}$ of benzidine $(0.005$ $\mathrm{mol})$ in $100 \mathrm{~mL}$ of glacial acetic acid, leads to the formation of $1.1 \mathrm{~g}$ of a brown crystal, 1,1'-diphenylenedipyrridyl (SchemeII), that is a $71 \%$ yield with $\mathrm{T}_{\mathrm{f}}>260{ }^{\circ} \mathrm{C}$.

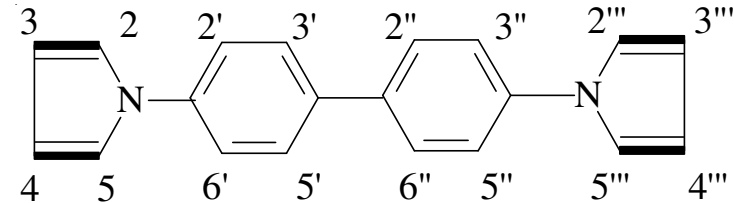

Scheme-II: Chemical formula of 1,1'-diphenylenedipyrridyl (DPhDPy)

${ }^{1} \mathrm{H}$ NMR (200 MHz, $\left.\mathrm{CDCl}_{3}\right): \delta 7.14($ app t, $J=2.1$ et 2.2 $\left.\mathrm{Hz}, 2 \mathrm{H}, \mathrm{H}_{2,5}\right), 6.38$ (app t, $J=2$ and $\left.2.1 \mathrm{~Hz}, 2 \mathrm{H}, \mathrm{H}_{3,4}\right), 7.48(\mathrm{~d}$, $\left.J=8.5 \mathrm{~Hz}, 2 \mathrm{H}, \mathrm{H}_{2^{\prime}, 6^{\prime}}\right), 7.68\left(\mathrm{~d}, J=8.4 \mathrm{~Hz}, 8 \mathrm{H}, \mathrm{H}_{3^{\prime}, 5^{\prime}}\right)$. FT-IR $\left(\mathrm{KBr}, \mathrm{cm}^{-1}\right): 3139 ; 3049 ; 3073 ; 3042 ; 1609 ; 1511 ; 1476 ; 1553$; $1408 ; 820 ; 1067 ; 730 ; 612 ; 516$. Elemental analysis $(\%)$ : Calculated: C: $84.48, \mathrm{H}: 5.67, \mathrm{~N}: 9.85$; obtained: C: $83.17, \mathrm{H}$ : $5.43, \mathrm{~N}: 9.52$.

Mechanism of the synthesis reaction: The synthesis of PhDPy and DPhDPy was realized according to the method proposed by Clauson-Kaas ${ }^{31}$ and as described in our previous paper $^{28}$. Scheme-III describes a proposed reaction mechanism for this synthesis. As indicated, the mechanism of this synthesis involves a succession of steps, beginning with a condensation reaction between $p$-substituted aniline and 2,5-dimethoxytetrahydrofurane in glacial acetic acid solution.

Then, the nucleophilic attack of the free nitrogen doublet of the amino group occurred on the 2 and 5 positions of 2,5dimethoxytetrahydrofuran, followed by a removal of both methoxy groups and an intramolecular dehydration between reaction intermediates leading to the final product. In case of $p$-phenylenediamine and benzidine condensation, we observed an immediate precipitation of the desired product, PhDPy or DPhDPy. Also, it is important to note that for PhDPy and DPhDPy synthesis, the yield is proportional to the number of amino sites transformed into pyrrolic derivatives ${ }^{28}$ (Table-1).

\section{TABLE-1}

SYNTHESIS YIELD OF $p$-SUBSTITUTED N-PhPy OBTAINED USING THE MODIFIED CLAUSON-KAAS METHOD

\begin{tabular}{cccc} 
Aromatic amine & Abbreviation & Chemical formulas & $\begin{array}{c}\text { Yield } \\
(\%)\end{array}$ \\
\hline$p$-Phenylenediamine & PhDPy & $\mathrm{C}_{14} \mathrm{H}_{12} \mathrm{~N}_{2}$ & $36^{\mathrm{a}}$ \\
Benzidine & DPhDPy & $\mathrm{C}_{20} \mathrm{H}_{16} \mathrm{~N}_{2}$ & 71 \\
\hline
\end{tabular}

${ }^{a}$ Yields of 15 and $41 \%$ previously obtained with the Clauson-Kaas method $^{28,31,32}$

UV-visible absorption spectroscopy of PhDPy and DPhDPy $^{28}$ : In case of these symmetric oligophenylenes possessing pyrrole groups on their extremities (PhDPy and DPhDPy), it appears, in the UV-visible absorption spectra in dimethylsulfoxide (DMSO) solution, a single, but very wide band around $277-278$ and 302-303 nm, respectively, with very high molar absorption coefficients $\left(\log \lambda_{\max }=4.26-5.14\right.$ and 4.82-4.93, respectively). This band corresponds to the ${ }^{1} \mathrm{~L}_{a} /{ }^{1} \mathrm{~L}_{b}$ $\pi-\pi^{*}$ electronic transition of the oligophenylene moiety (aromatic system), as previously indicated ${ }^{28}$. As can be seen in Fig. 1, the absorption maximum of this transition undergoes a considerable bathochromic shift $\left(\Delta \lambda_{\mathrm{A}}=25 \mathrm{~nm}\right)$ from the spectrum of PhDPy to that of DPhDPy, whereas a comparable hypsochromic shift is observed from the spectrum of PhDPy to that of $\mathrm{N}$-phenylpyrrole ${ }^{27}$. On the other hand, the value of
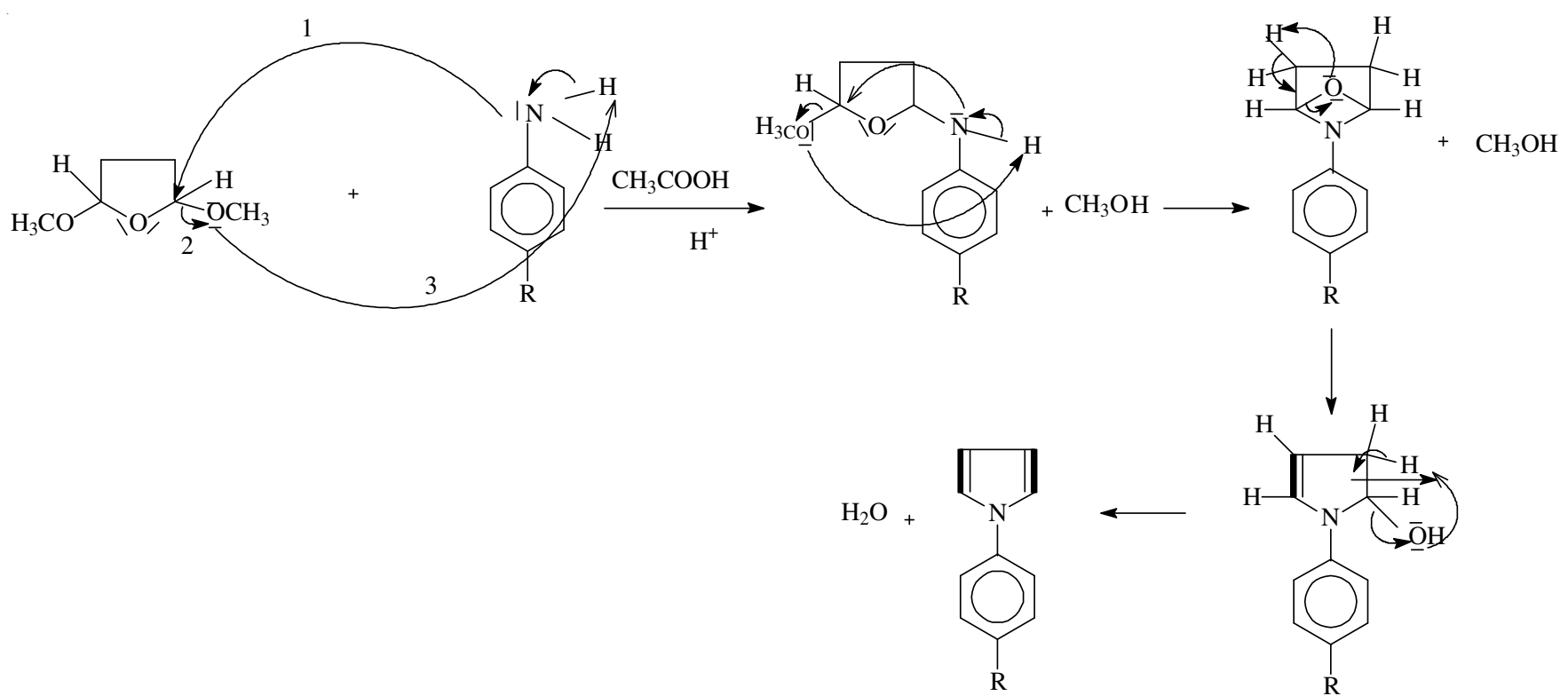

Scheme-III: Proposed multi-step synthesis mechanism of $p$-substituted $\mathrm{N}$-phenylpyrroles. The synthesis followed $\mathrm{Clauson}^{-\mathrm{Kaa}}$ method $\left(\mathrm{R}=\mathrm{NH} \mathrm{H}_{2}\right.$ or phenylamine) 


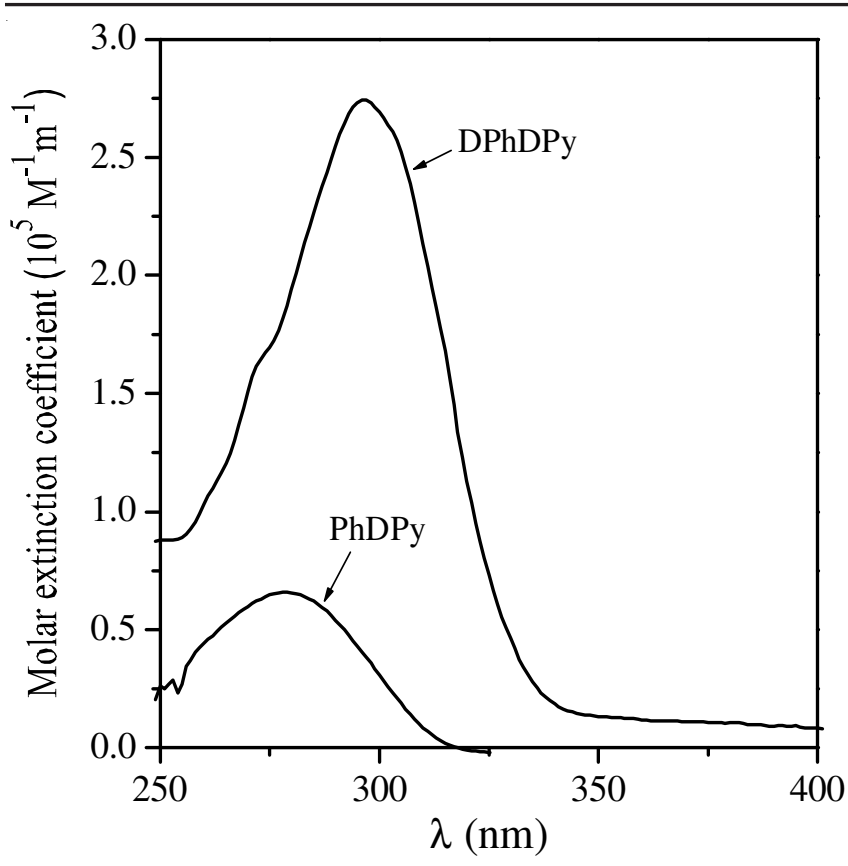

Fig. 1. UV-visible absorption spectra of PhDPy $\left(10^{-5} \mathrm{M}\right)$ and DPhDPy $\left(10^{-6}\right.$ M) in DMSO at room temperature ${ }^{28}$

$\varepsilon_{\max }$ rises as the oligophenylene chain length is increased of one or two phenyl units. These spectral changes can be attributed to the extension of the conjugated aromatic system ${ }^{28}$.

Monomer electro-oxidation: The study of the electrochemical characteristics of $10^{-5} \mathrm{M}$ PhDPy and DPhDPy acetonitrile solution containing $10^{-1} \mathrm{M} \mathrm{Bu}_{4} \mathrm{NPF}_{6}$, was realized by cyclic voltammetry $(\mathrm{CV})$ between 0 and $1.5 \mathrm{~V} / \mathrm{ECS}$ at scan rates of 500, 200, 100 and $50 \mathrm{mVs}^{-1}$. The voltammograms of the PhDPy oxidation (Fig. 2) show two irreversible anodic waves at oxidation potential values of 0.85 and $1.25 \mathrm{~V} / \mathrm{ECS}$ which can be assigned, respectively, to the oxidation of the pyrrole and phenyl groups into radical-cation. In the case of DPhDPy, these anodic waves appear around 0.84 and 1.20 V/ECS. The PhDPy oxidation potentials are lowered by about $250 \mathrm{mV}$ relatively to $\mathrm{N}-\mathrm{PhPy}$ in the absence of pyrrole and of about $150 \mathrm{mV}$ with regard to N-PhPy in the presence of small amounts of pyrrole ${ }^{26,27}$. The PhDPy potential lowering, observed by comparison to un-substituted N-PhPy and pyrrole-electrocatalyzed N-PhPy oxidation, seems directly related to the increase of chain length resulting from the extension of the electronic $\pi$-conjugated system, induced by the presence of a second pyrrole group. This potential decrease is thus clearly attributable to the extension of the electronic $\pi$-conjugated system rather than to the electrocatalytic effect of a monomer with a lower oxidation potential. The oxidation peaks current density (j) values increase linearly with the scan rate (Fig. 2). The electrochemical process is thus controlled by the chemical reaction occurring on the electrode surface. In contrast, in the case of N-PhPy, the oxidation process was controlled by the diffusion of the electroactive species in the solution, since the peak current was proportional to the scan rate square root ${ }^{26,27}$. This difference of behaviour in radical-cation formation reaction kinetics could be related to the size of the studied monomer.

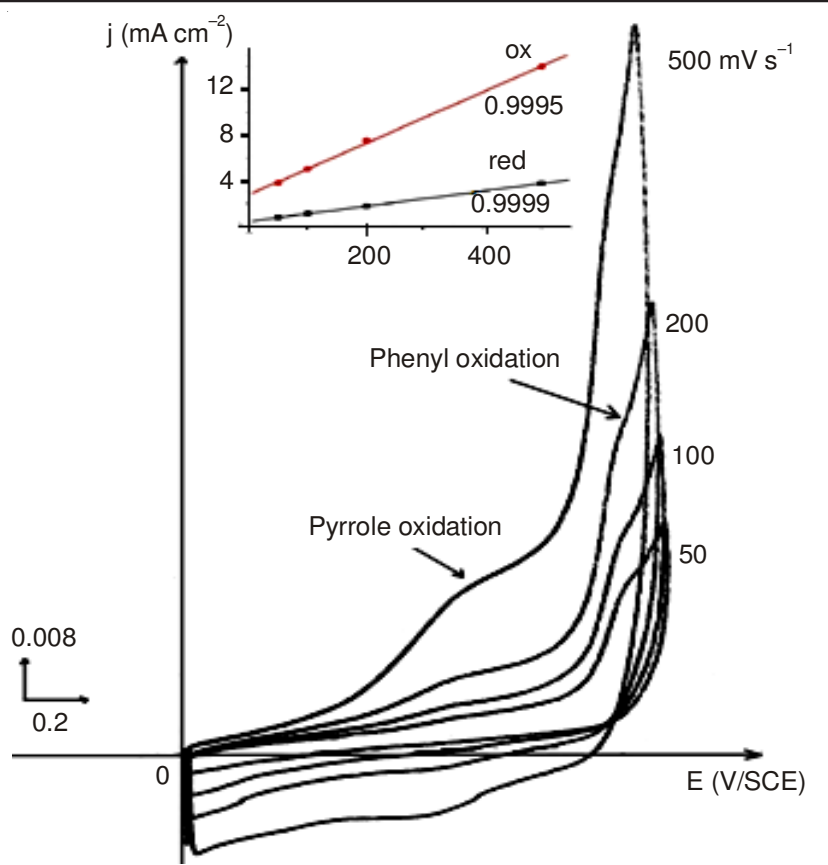

Fig. 2. Voltammograms of PhDPy electro-oxidation on Pt electrode in an acetonitrile solution of $10^{-5} \mathrm{M} \mathrm{PhDPy}+0.1 \mathrm{M} \mathrm{Bu}_{4} \mathrm{NPF}_{6}$ at different scan rates. Insert: $\mathrm{j} v s$. scan rate in oxidation and reduction processes with the corresponding adj. $\mathrm{R}^{2}$ values

\section{Electropolymerization of $1,1^{\prime}$-phenylenedipyrridyl and 1,1'-diphenylenedipyrridyl}

Electropolymerization: We achieved the electropolymerization of PhDPy by performing ten potential scanning cycles between 0 and $1.3 \mathrm{~V} / \mathrm{ECS}$ at $100 \mathrm{mV} \mathrm{s}^{-1}$ in an acetonitrile solution containing $10^{-2} \mathrm{M}$ of $\mathrm{PhDPy}$ and $0.1 \mathrm{M}^{\circ} \mathrm{Bu}_{4} \mathrm{NPF}_{6}{ }^{30}$. We noted the formation of reddish, electroactive poly(PhDPy) films on the Pt electrode. The obtained cyclic voltammograms (CV) (Fig. 3) show a rather wide anodic peak towards $0.55 \mathrm{~V} /$ ECS as well as a wide cathodic peak towards $0.45 \mathrm{~V} / \mathrm{ECS}$. The anodic peak can be attributed to the formation of oligomers having various sizes, whereas the cathodic peak corresponds to the reduction the same oligomers. As the electropolymerization progresses, the current densities of these peaks regularly increase with the number of cycles, indicating the progressive growth of the poly( $\mathrm{PhDPy})$ film formed on electrode surface. In addition, we observed a decrease of about $200 \mathrm{mV}$ in the polymer oxidation and reduction potentials relatively to poly(N-PhPy) and of about $100 \mathrm{mV}$ with regard to poly(NPhPy) electrocatalyzed by pyrrole ${ }^{27,30}$. This decrease of poly (PhDPy) oxidation and reduction potentials in comparison with poly (N-PhPy) is assigned to the conjugation effects resulting from the presence of a second pyrrolyl group in para position on the phenyl ring, causing the extension of the $\pi$-conjugated system in the polymer structure ${ }^{30}$.

To electropolymerize DPhDPy, we applied the same CV method, but, because of the low solubility of this monomer in acetonitrile, we used a solution containing $10^{-3} \mathrm{M}$ of DPhDPy (saturated solution) and $0.1 \mathrm{M}$ of $\mathrm{Bu}_{4} \mathrm{NPF}_{6}$ in dichloromethane. The resulting voltammograms are characterized by broad anodic and cathodic waves, appearing near 0.75 and $0.40 \mathrm{~V} / \mathrm{ECS}$, respectively. The current densities of these peaks increaseweakly with the number of cycles, which corresponds to a 


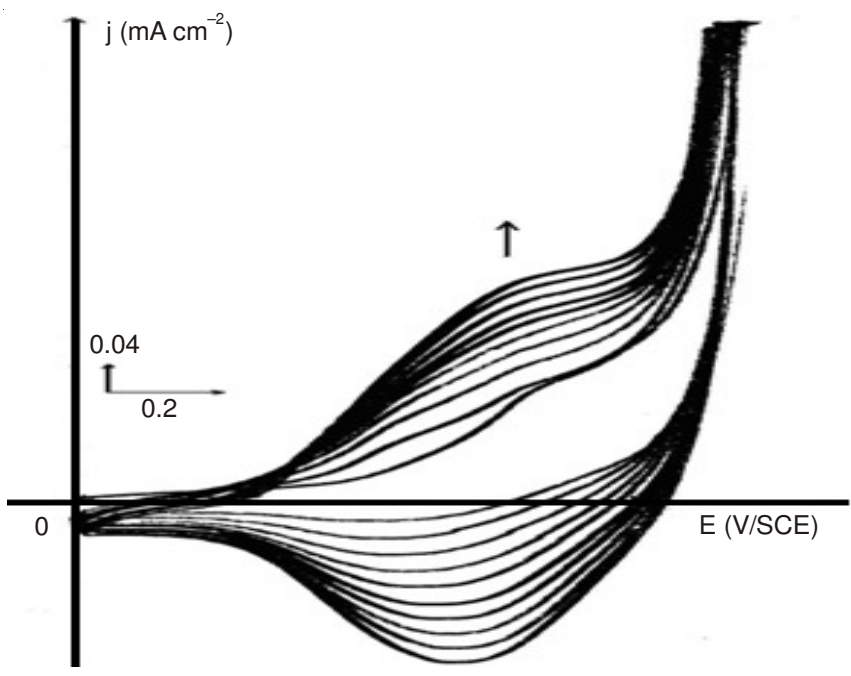

Fig. 3. Voltammograms of poly (PhDPy) film electrodeposition on $\mathrm{Pt}$ electrode in an acetonitrile solution of $10^{-2} \mathrm{M} \mathrm{PhDPy}+0.1 \mathrm{M}$ $\mathrm{Bu}_{4} \mathrm{NPF}_{6}$. Scan rate: $100 \mathrm{~m} \mathrm{Vs}^{-1}$

progressive growth of a grayish and very thin poly(DPhDPy) film on the Pt electrode. The deposition of very thin polymer films as well as the relatively high values of the poly(DPhDPy) oxidation potential compared with that of poly(PhDPy) could be explained by the formation of oligomers of shorter pyrrolic chains, resulting from the increase of the size of the interchain bridge which would produce a steric effect, as already previously noted $^{30}$. The same reason could explain the very weak decrease of the poly(DPhDPy) oxidation potential in comparison with that of the monomer ( $\triangle \mathrm{E} 100 \mathrm{mV} / \mathrm{ECS})$.

Electrosynthesis in potentiostatic and galvanostatic modes: We also successfully carried out the electropolymerization of PhDPy and DPhDPy in the galvanostatic mode. The obtained chronopotentiometric curves are depicted in Figs. $4 \mathrm{a}$ and $4 \mathrm{~b}$. After 5 min of polarization, good-quality films are obtained, for current density values comprised between 0.125 and $0.5 \mathrm{~mA} \mathrm{~cm}^{-2}$ for poly(PhDPy) films and between 0.125 and $0.25 \mathrm{~mA} \mathrm{~cm}^{-2}$ in the case of poly(DPhDPy) films. For $\mathrm{j}=$ $0.5 \mathrm{~mA} \mathrm{~cm}^{-2}$, we noted in the case of poly(DPhDPy), the appearance of a second plateau towards 1.6 V/ECS after a polarization time of $30 \mathrm{sec}$. As films obtained in these conditions are of poor quality, this plateau is probably due to a degradation resulting from the polymer overoxidation. For a $\mathrm{j}$ value of $1 \mathrm{~mA} \mathrm{~cm}$, this second plateau gradually appears after approximately $5 \mathrm{sec}$ for DPhDPy, 2 min for PhDPy and $4 \mathrm{~min}$ for $\mathrm{NPhPy}$. This could explain the easier electropolymerization of N-PhPy. However, these results prove to be somewhat dissimilar in both cases studied and could be attributed to a steric effect related to the electropolymerization double-site resulting from the presence of both terminal pyrroles. Moreover, this steric hindrance of the electropolymerization process could explain, at least in the case of poly(DPhDPy), the very thin films obtained by cyclo-voltammogram ${ }^{30}$.

The potentiostatic mode was also applied to electropolymerize PhDPy and DPhDPy. The nucleation phase appears almost immediately with curves in the form of plateaus resulting in a constant electrosynthesis current corresponding to the deposition of an electroactive film. Similar-results have already been found in the case of N-PhPy in acidified direct

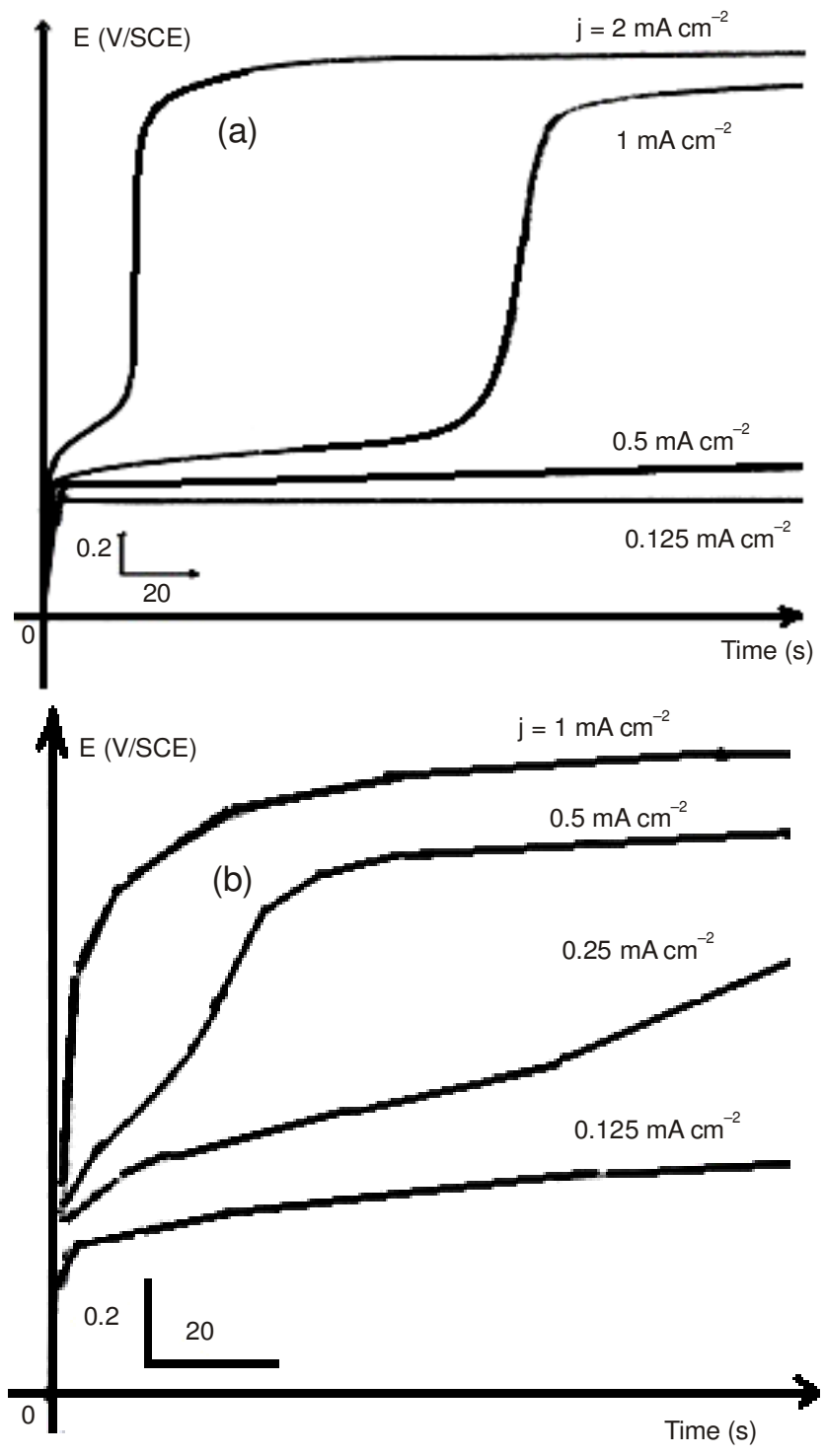

Fig. 4. Chronopotentiometric curves for the poly(PhDPy) (a) and (b) poly(DPhDPy) film electrodeposition on Pt electrode, performed in acetonitrile solution of $10^{-2} \mathrm{M} \mathrm{PhDPy}+0.1 \mathrm{M} \mathrm{Bu}_{4} \mathrm{NPF}_{6}$ (a) and in dichloromethane solutions of $10^{-3} \mathrm{M} \mathrm{DPhDPy}+0.1 \mathrm{M}$ $\mathrm{Bu}_{4} \mathrm{NPF}_{6}(\mathrm{~b})$

micellar medium ${ }^{27,29}$. For these compounds, the electrodeposition potential increases (in the domain in which good quality films are formed) with the current density during the electropolymerization process and then it remains virtually stable which indicates that the films remain fairly conductive.

\section{Characterization of the films}

Electrochemical properties: We studied the electroactivity of the polymers electrodeposited on the Pt electrode in acetonitrile in the presence of $\mathrm{Bu}_{4} \mathrm{NPF}_{6} 0.1 \mathrm{M}$ by varying the number of cycles (Fig. 5a) and the scan rate (Fig. 5b). The films are stable after twelve cycles with a loss of electroactivity of about $7 \%$ (against $13 \%$ for poly $(\mathrm{N}-\mathrm{PhPy})^{26}$ ). Fig. $5 \mathrm{~b}$ shows an anodic peak at $0.55 \mathrm{~V} / \mathrm{ECS}$ and a cathodic one at $0.45 \mathrm{~V} /$ ECS corresponding, respectively, to the oxidation and to the reduction of poly(PhDPy). The intensity of this redox wave increases with the scan rate. The study of the doping kinetics shows a linear evolution of the intensity of these peaks with 

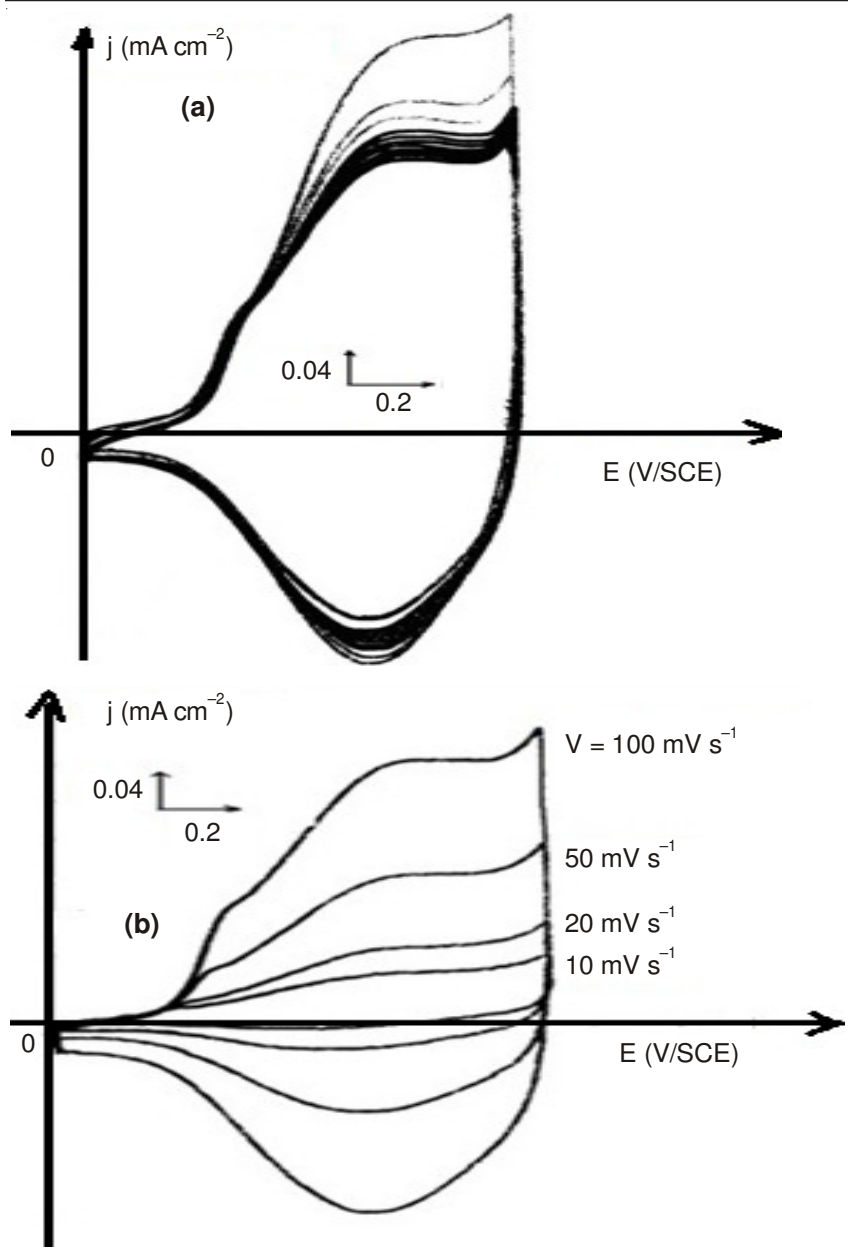

Fig. 5. Electroactivity of poly(PhDPy) films electrodeposited on the $\mathrm{Pt}$ electrode in acetonitrile in the presence of $\mathrm{Bu}_{4} \mathrm{NPF}_{6} 0.1 \mathrm{M}$, at $\mathrm{v}=$ $100 \mathrm{mVs}^{-1}$ (different number of cycles) (a) and at various scan rates (b)

the scan rate, which suggests an electrochemical process controlled by reactions at the electrode surface, as previously observed $^{30}$. It is also noted that electrochromic effect which is evidenced by a change of color from the oxidized form (red) to the reduced form (green). The poly(PhDPy) oxidation and reduction potential values undergo a decrease of about 200 $\mathrm{mV}$ compared with poly(N-PhPy) and of $100 \mathrm{mV}$ in the presence of small amounts of pyrrole $\mathrm{e}^{26,27}$. This confirms an improvement of the electrochemical properties of the polymer and a better stability when a second pyrrole ring is directly grafted on $\mathrm{N}$ phenylpyrrole.

FT-IR spectroscopy: We recorded the FT-IR spectra of poly (PhDPy) films, in the oxidized and reduced forms. The reduction was chemically carried out in a $10 \%$ (wt) ammonia solution on films electrosynthesized by $\mathrm{CV}$ (linear potentialscan between 0.0 and $1.3 \mathrm{~V} / \mathrm{ECS}$ during 10 cycles at $100 \mathrm{mV} \mathrm{s}^{-1}$ on Pt-coated glass plates). The FT-IR data of the studied poly (PhDPy), of the corresponding monomer and of N-PhPy for comparison purposes, as well as the attributions of the main IR bands, are presented in Table-2. For the assignments of the bands, we compared our IR data with previous works on analogous compounds such as un-substituted and substituted $\mathrm{N}$-PhPys and their respective polymers ${ }^{26,27,29,30,33-35}$. The comparative study of the polymers and corresponding monomers, in particular the analysis of the polymerization-induced modifications (bands disappearance, shifts or intensity variation) gave information about the coupling sites of the polymer chains.

The IR spectrum of PhDPy shows two very intense bands in the $715-730 \mathrm{~cm}^{-1}$ region, attributed to the out-of-plane $\gamma(\mathrm{C}-\mathrm{H})$ deformation vibrations of the pyrrole group $(4 \mathrm{H})$. These bands disappear completely or are considerably reduced and shifted in the corresponding polymer spectra, which indicates that the electropolymerization takes place only on the pyrrolic ring. Strong bands appearing at $1068-1023 \mathrm{~cm}^{-1}$ in the PhDPy spectrum are assigned to $\gamma \mathrm{C}$-H deformation vibration modes of two hydrogen atoms in the a position of N-substituted Py. It is interesting to note that these bands disappear or decrease in intensity in the poly(PhDPy) spectra, which shows that the coupling takes place in the 2 and 5 positions of the pyrrolic ring, as previously noted ${ }^{30}$. Concomitantly, a new band occurs at $980 \mathrm{~cm}^{-1}$ in the polymer spectra only, which is characteristic of the $\mathrm{C}-\mathrm{H}$ bond out-of-plane deformation vibration of a 1,2,5tri-substituted pyrrole $27,29,30$.

In addition, other bands clearly appear at $875-840 \mathrm{~cm}^{-1}$ in the monomer spectrum and are weakly shifted at $862-834 \mathrm{~cm}^{-1}$, in the reduced polymer spectrum, which correspond to the $\gamma(\mathrm{C}-\mathrm{H})$ out-of-plane deformation vibrations of four adjacent hydrogen atoms of the 1,4-disubstituted phenyl groups. This confirms that both substituents in para position on the monomer phenyl group are still present in the corresponding polymer and that no elimination reaction occurs on the phenyl ring during the coupling electropolymerization process. All these IR spectral results indicate that the chain extension during the $\mathrm{PhDPy}$ electropolymerization takes only place by coupling reactions in the 2 and 5 positions of the pyrrolic units ${ }^{30}$.

TABLE-2

IR WAVENUMBERS $\left(\mathrm{cm}^{-1}\right)$ AND ASSIGNMENTS OF THE MAIN

BANDS OF NPhPy AND PhDPy (IN KBr Pellets) AND OF REDUCED poly(PhDPy) ON Pt-COATED GLASS PLATES ${ }^{26-30}$

\begin{tabular}{|c|c|c|c|}
\hline $\mathrm{N}-\mathrm{PhPy}$ & PhDPy & Poly(PhDPy) & Assignment \\
\hline $\begin{array}{l}3151 ; 3141 \\
3113 ; 3102\end{array}$ & $3125 ; 3109$ & 3104 & $v(\mathrm{C}-\mathrm{H})$ stretching $(\mathrm{Py})$ \\
\hline $\begin{array}{l}3063 ; 3046 \\
3019\end{array}$ & $3073 ; 3047$ & 3050 & $v(\mathrm{C}-\mathrm{H})$ stretching $(\mathrm{Ph})$ \\
\hline 1694 & - & 1716 & $v(\mathrm{C}=\mathrm{O})$ \\
\hline $1604 ; 1591$ & 1577 & 1575 & $v(\mathrm{C}=\mathrm{C})$ stretching $(\mathrm{Ph})$ \\
\hline 1513 & $1526 ; 1486$ & 1529 & $v(\mathrm{C}=\mathrm{C})$ stretching $(\mathrm{Ph})$ \\
\hline $\begin{array}{l}1556 ; 1470 ; \\
1460 ; \\
1401\end{array}$ & $\begin{array}{l}\text { 1472; 1433; } \\
1388\end{array}$ & $\begin{array}{l}1475 ; 1460 \\
1402 ; 1388\end{array}$ & $v(\mathrm{C}=\mathrm{C}) ; \mathrm{v}(\mathrm{C}-\mathrm{N})(\mathrm{Py})$ \\
\hline $\begin{array}{l}1255 ; 1189 ; \\
1164\end{array}$ & $1257 ; 1244$ & $1243 ; 1165$ & $\begin{array}{l}\delta(\mathrm{C}-\mathrm{H}) \text { in-plane }(\mathrm{Ph}) 4 \mathrm{H}(1,4- \\
\text { disub-benzene })\end{array}$ \\
\hline 1071 & $1068 ; 1023$ & $1068 ; 1034$ & $\gamma\left(\mathrm{C}-\mathrm{H}_{\alpha}\right)(\mathrm{Py}) 4 \mathrm{H}$ \\
\hline- & - & 980 & $v(\mathrm{C}-\mathrm{H})(1,2,5$-trisub-Py $)$ \\
\hline $\begin{array}{l}896 ; 868 \\
759 ; 688\end{array}$ & $875 ; 840$ & $862 ; 834$ & $\begin{array}{l}\gamma(\mathrm{C}-\mathrm{H}) \text { out-of-plane bending } \\
(\mathrm{Ph}) 4 \mathrm{H}(1,4-\text { disub-benz })\end{array}$ \\
\hline $1016 ; 720$ & $727 ; 714$ & $720 ; 714$ & $\begin{array}{l}\gamma(\mathrm{C}-\mathrm{H}) \text { out-of-plane bending } \\
\text { (Py) } 4 \mathrm{H}\end{array}$ \\
\hline
\end{tabular}

Also, the PhDPy and the corresponding polymer IR spectra show very close bands in the $1577-1575$ and $1529-1486 \mathrm{~cm}^{-1}$ regions. These bands are ascribed to $v(C=C)$ stretching vibrations of phenyl groups, but their intensity and number significantly varies upon passing from the monomer to the 
polymer spectra. These differences between the monomer and the poly(PhDPy) IR spectra could be due to the existence of variable interactions between the para-substituted phenyl groups and the polypyrrole chain in the polymer, as well as to the increase of conjugation in the para-substituted phenyl groups, resulting from the presence of a relatively long $\mathrm{N}$-substituted polypyrrole chain ${ }^{30}$.

X-photoelectron spectroscopy: The XPS analysis of poly(PhDPy) films obtained in the oxidized and reduced states by CV (10 cycles) shows the presence of carbon, nitrogen, fluorine and phosphor atoms and confirms the formation of a $\mathrm{PF}_{6}{ }^{-}$-doped poly(PhDPy) film. As found in previous paper $^{30}$, the doping level, calculated from the ratio $\mathrm{P} / \mathrm{N} \%$, is close to $30 \%$ in the oxidized state and $7 \%$ in the reduced state. These doping level values are very similar to those described in the case of other pyrrole derivatives ${ }^{26,27,30,37-39}$. The deconvolution of the carbon and nitrogen signals provides interesting features about the films structure.

In the case of poly(PhDPy) and poly(DPhDPy), the peakfitted $\mathrm{C} 1 \mathrm{~s}$ spectra show, after deconvolution, the presence of two components (Figs. $6 \mathrm{a}$ and $6 \mathrm{~b}$ ). The most intense component is located at $285 \mathrm{eV}$ and corresponds to the $\mathrm{C}-\mathrm{C}$ and $\mathrm{C}=\mathrm{C}$ aliphatic and aromatic bonds of the polymer skeletons $s^{30,37,38}$. The second, weaker component, appearing at around $286 \mathrm{eV}$, is attributed to the pyrrole $\mathrm{C}-\mathrm{N}(\alpha-\mathrm{C})$ bond $^{30,37,38}$.

The N1s signal of the oxidized and reduced poly( $\mathrm{PhDPy})$ film spectra exhibits a component near $400 \mathrm{eV}$, which is due to the neutral nitrogen of the pyrrole ring $(\mathrm{C}-\mathrm{N})$ and a second component at about $402 \mathrm{eV}$, corresponding to a positively charged $\mathrm{N}^{+}$of the pyrrole ring $\left(\mathrm{C}-\mathrm{N}^{+}\right)^{30}$. The latter component intensity in the polymer increases, when going from the reduced to the oxidized state. Moreover, the polymer reduction corresponds to a release of $\mathrm{PF}_{6}{ }^{-}$anions, which is confirmed by a decrease of the $\mathrm{P} / \mathrm{N}$ ratio for the reduced poly $(\mathrm{PhDPy})$ film. Also, we noted an increase of the $\mathrm{N}^{+} / \mathrm{C}$ ratio for the poly (PhDPy) oxidized state, which could be due to a reorganization of the bonds (electronic redistribution) within the oxidized polymer produced by the entrance of $\mathrm{PF}_{6}{ }^{-}$anions in the film.

Scanning electronic microscopy: The SEM microphotos of oxidized and reduced forms of poly(PhDPy) (Figs. 7a and 7b) obtained in the galvanostatic mode by applying a current density of $0.125 \mathrm{~mA} \mathrm{~cm}^{-2}$ during $5 \mathrm{~min}$ show an uneven electrode surface covered by homogeneous films with regular aggregates. The passage from the oxidized state to the reduced one comes along with an evolution of the morphology from a relatively smooth surface with aggregates to a rough and vague surface $^{30}$. These poly(PhDPy) SEM photos are very close to those described in the case of N-phenylpyrrole and other substituted pyrroles ${ }^{17,23,30}$.

UV-visible absorption spectra: The solid-state UV-visible absorption spectra of poly(PhDPy) films (Fig. 8) in the oxidized and reduced states, electrodeposited on ITO plaques at $\mathrm{E}=1$. $3 \mathrm{~V} / \mathrm{ECS}$ during $2 \mathrm{~min}$, reveal a very intense absorption band at about $362 \mathrm{~nm}$ (located at $347 \mathrm{~nm}$ for un-substituted poly $(\mathrm{N}-$ PhPy) film, electrosynthesized in acetonitrile $\left.{ }^{26}\right)$. This band, of which the intensity increases upon going from the oxidized state to the reduced state, is attributed to a delocalized $\pi-\pi^{*}$ electronic transition in the polymer film ${ }^{26}$. A second, very wide band appears, only in the oxidized form spectrum, between
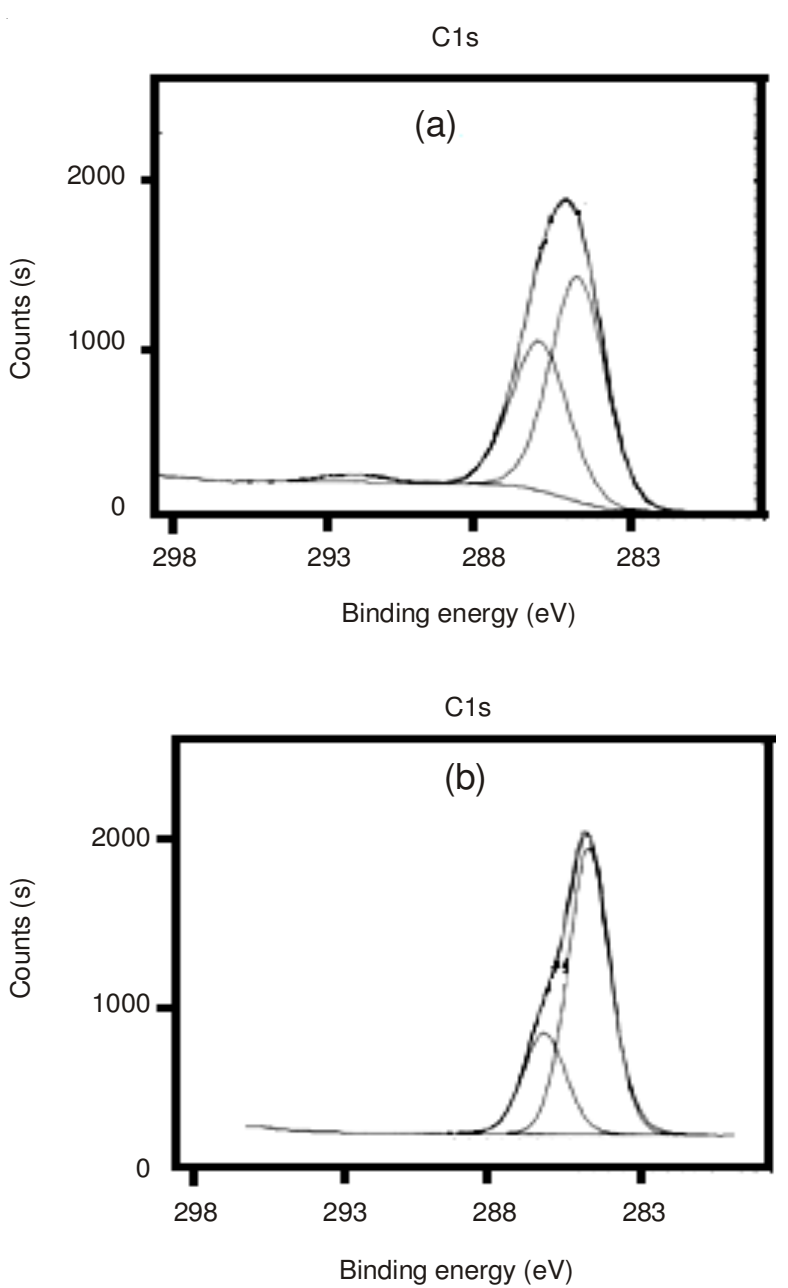

Fig. 6. Carbon XPS deconvolution peaks of poly(PhDPy) (a) and poly(DPhDPy) (b)
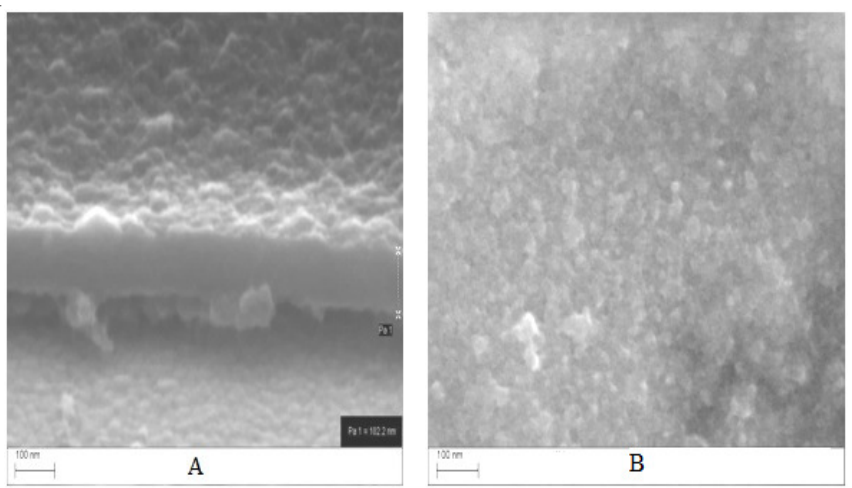

Fig. 7. SEM photos of oxidized (a) and reduced (b) poly(PhDPy) films obtained on ITO plaques with $\mathrm{J}=0.5 \mathrm{~mA} / \mathrm{cm}^{2}$ during $5 \mathrm{~min}$

480 and $573 \mathrm{~nm}$ and could be related to a $\pi-\pi^{*}$ electronic transition in the polymer conjugated cationic segments ${ }^{26,37}$. The redshift of about $15 \mathrm{~nm}$ of the poly(PhDPy) absorption maximum relative to un-substituted poly $(\mathrm{N}-\mathrm{PhPy})^{26}$ was attributed to the electron-donating effect of the second pyrrole group in para position on the phenyl ring, which increased the conjugation of the polymer aromatic system. Also, a stronger red-shift (85 $\mathrm{nm}$ ), noted upon going from the PhDPy spectrum to the corresponding polymer spectrum, might partly result from the conjugation increase in the polymer, due to the $\pi-\pi^{*}$ electronic transition delocalization. 


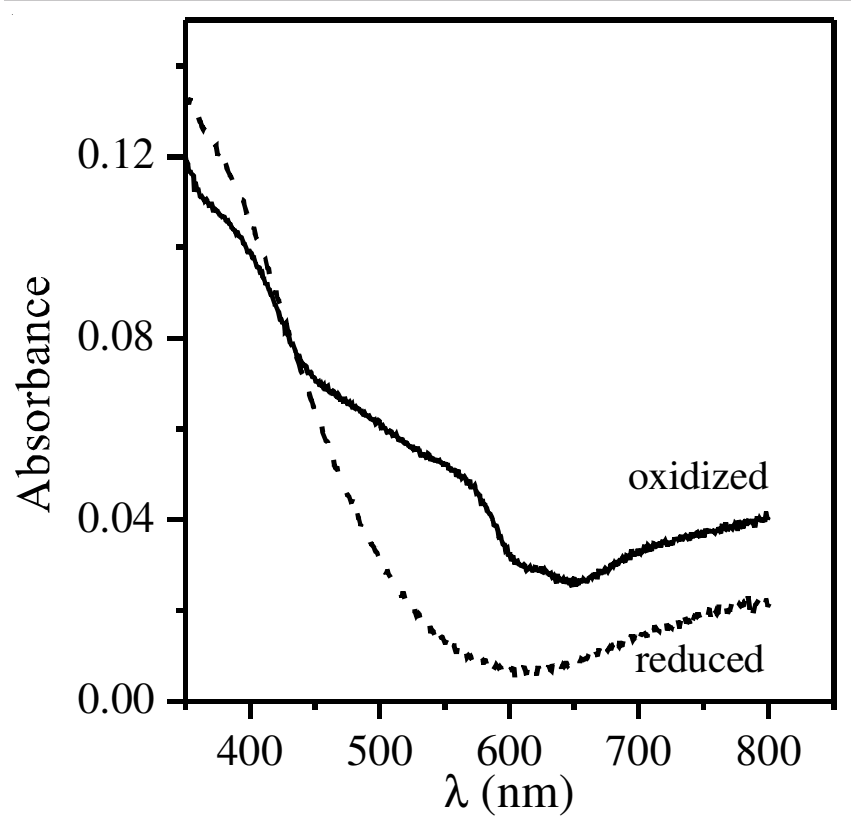

Fig. 8. Solid-state UV-visible absorption spectra of oxidized and reduced poly(PhDPy) electrodeposited on ITO glass at $\mathrm{E}=1.3 \mathrm{~V} / \mathrm{ECS}$ during $2 \mathrm{~min}$

\section{Conclusion}

The organic synthesis of 1,1'-phenylenedipyrridyl (PhDPy) and 1,1'-diphenylenedipyrridyl (DPhDPy) was successfully performed with relatively satisfactory yields (35 and $71 \%$ ). The pure compounds with interesting physico-chemical and optical characteristics were obtained. The extension of the $\pi$-system by grafting of pyrrole modifies the monomer oxidation kinetics, the reaction on the electrode surface becoming the limiting step, which is not the case during the pyrroleelectrocatalyzed oxidation of un-substituted $\mathrm{N}-\mathrm{PhPy}$. The electropolymerization of PhDPy leads to the formation of adherent and electroactive poly (PhDPy) films having properties clearly better than those of poly (N-PhPy) films, obtained by pyrrole-electrocatalyzed electropolymerization. Therefore, the extension of the electronic $\pi$-system by grafting of conjugated substituents, such as pyrrole group(s), has more positive effects on the improvement of the polymer physicochemical properties than those resulting from the addition of small amounts of pyrrole in the electrosynthesis medium (electrocatalytic effect). However, this modeling or improvement of the physicochemical properties of a polymer realized by extending the monomer electronic $\pi$-system presents its limits when electropolymerizing a larger size monomer, like DPhDPy. Indeed, in this case, a very slow and weak growth is observed for poly (DPhDPy) films which also possess physicochemical properties poorer than those of poly $(\mathrm{N}-\mathrm{PhPy})$. These constraints are probably due to steric effects which are in opposition to the mesomeric effect, thus reducing the solubility and limiting the polymerization to the formation of short-chain oligomers.

\section{REFERENCES}

1. Y. Hayakawa, K. Kawakami, H. Seto and K. Furihata, Tetrahedron Lett., 33, 2701 (1992).

2. W.Y. Yoshida, K.K. Lee, A.R. Carroll and P.J. Scheuer, Helv. Chim. Acta, 75, 1721 (1992).

3. A. Jones, Pyrroles, Wiley, New York (1990).

4. R. Jackstell, H. Klein, M. Beller, K.-D. Wiese and D. Röttger, Eur. J. Org. Chem., 2001, 3871 (2001).

5. J. Lehuédé, B. Fauconneau, L. Barrier, M. Ourakow, A. Piriou and J.M. Vierfond, Eur. J. Med. Chem., 34, 991 (1999).

6. J. Arrowsmith, S.A. Jennings, A.S. Clark and M.F.G. Stevens, J. Med. Chem., 45, 5458 (2002).

7. A. Hantzsch, Ber. Dtsch. Chem. Ges, 23, 1474 (1890).

8. D.C. van Beelen, J. Wolters and A. van der Gen, Recl. Trav. Chim. Pays Bas, 98, 437 (1979).

9. V.F. Ferreira, M.C.B.V. De Souza, A.C. Cunha, L.O.R. Pereira and M.L.G. Ferreira, Org. Prep. Proced. Int., 33, 411 (2001).

10. L. Knorr, Ber. Dtsch. Chem. Ges, 17, 1635 (1884).

11. J. M. Hamby and J. C. Hodges, Heterocycles, 35, 843 (1993).

12. I. Elghamry, Synth. Commun., 32, 897 (2002).

13. C. Paal, Ber. Dtsch. Chem. Ges., 17, 2756 (1884).

14. P.-K. Chiu, K.-H. Lui, P.N. Maini and M.P. Sammes, J. Chem. Soc. Chem. Commun., 109 (1987).

15. C. Pak-Kan and S. Michael P, Tetrahedron, 46, 3439 (1990).

16. K.A. Bunten and A.K. Kakkar, Macromolecules, 29, 2885 (1996).

17. J. Nakayama, H. Dong, K. Sawada, A. Ishii and S. Kumakura, Tetrahedron, 52, 471 (1996).

18. T.E. Moustafid, S. Aeiyach, J.J. Aaron, H. Mir-Hedayatullah and P.C. Lacaze, Polymer, 32, 2461 (1991).

19. X.-C. Li, H. Sirringhaus, F. Garnier, A.B. Holmes, S.C. Moratti, N. Feeder, W. Clegg, S.J. Teat and R.H. Friend, J. Am. Chem. Soc., 120, 2206 (1998).

20. M.E. Nicho, H. Hu, C.L. Mata and J. Escalante, Sol. Energy Mater. Sol. Cells, 82, 105 (2004).

21. C. Taliani, R. Zamboni, R. Danieli, P. Ostoja, W. Porzio, R. Lazzaroni and J.L. Bredas, Phys. Scr., 40, 781 (1989).

22. D. Gningue-Sall, M. Fall, M.M. Dieng, J.J. Aaron and P.C. Lacaze, Phys. Chem. Chem. Phys., 1, 1731 (1999).

23. B. Grunden and J.O. Iroh, Polymer, 36, 559 (1995).

24. J.J.M. Halls, C.A. Walsh, N.C. Greenham, E.A. Marseglia, R.H. Friend, S.C. Moratti and A.B. Holmes, Nature, 376, 498 (1995).

25. K. Naoi, Y. Oura and M. Maeda, J. Electrochem. Soc., 142, 417 (1995).

26. A.K.D. Diaw, D. Gningue-Sall, A. Koné, G. Dione and M.M. Dieng, J. Soc. Ouest-Afr. Chim, 21, 77 (2006).

27. A.K.D. Diaw, D. Gningue-Sall, A. Koné and M.M. Dieng, J. Soc. OuestAfr. Chim., 24, 7 (2007).

28. A.K.D. Diaw, A. Yassar, D. Gningue-Sall and J.J. Aaron, ARKIVOC, xvii, 122 (2008).

29. A.K.D. Diaw, D. Gningue-Sall and J.-J. Aaron, Synth. Met., 161, 1483 (2011).

30. A.K.D. Diaw, D. Gningue-Sall, A. Yassar and J.-J. Aaron, Synth. Met., 179, 74 (2013).

31. N. Elming, N. Clauson-Kaas, M. Rottenberg, E. Stenhagen and S. Östling, Acta Chem. Scand., 6, 867 (1952).

32. P.-E. Just, K.I. Chane-Ching, J.C. Lacroix and P.C. Lacaze, J. Electroanal. Chem., 479, 3 (1999).

33. G. Socrate, Infrared Characteristic Group Frequencies, WileyInterscience Publication by John Wiley \& Sons New York (1980).

34. C. Mangeney, P.-E. Just, J.-C. Lacroix, K.I. Chane Ching, M. Jouini, S. Aeiyach and P.-C. Lacaze, Synth. Met., 102, 1315 (1999).

35. C. Mangeney, J.C. Lacroix, S. Aeiyach, M. Jouini, K.I. Chane-Ching and P.C. Lacaze, J. Chim. Phys., 95, 1535 (1998).

36. C.A. Ferreira, S. Aeiyach, M. Delamar and P.C. Lacaze, J. Electroanal. Chem., 284, 351 (1990).

37. D. Gningue-Sall, A. Kone, J.-J. Aaron, S. Aeiyach, M. Hedayatullah and P.-C. Lacaze, Synth. Met., 82, 119 (1996).

38. K. Boukerma, M. Omastová, P. Fedorko and M.M. Chehimi, Appl. Surf. Sci., 249, 303 (2005).

39. W. Prissanaroon, N. Brack, P.J. Pigram and J. Liesegang, Synth. Met., 142, 25 (2004). 